\title{
On the origin and invariance of the genetic assignments as elementary semiotic controls: A basic hypothesis
}

\author{
András Balázs \\ Department of Biological Physics, ELTE TTK Budapest, H - 1117 Pázmány s. 1/A, HUNGARY \\ Email address: \\ balazsandras2002@yahoo.com \\ To cite this article: \\ András Balázs. On the Origin and Invariance of the Genetic Assignments as Elementary Semiotic Controls: A Basic Hypothesis. \\ European Journal of Biophysics. Vol. 2, No. 3, 2014, pp. 17-28. doi: 10.11648/j.ejb.20140203.12
}

\begin{abstract}
In this paper we hope to place the two basic facts of life, constant active self - maintenance and self reproduction, into a fresh light. The basic idea is considering the genetic assignments as a „biological invariant of motion”, the latter forming a hierarchically produced, „,self - constructing”, regressive time inversion symmetry restoration, a closed semantic real - time upbuilding loop. It is, actually, the predetermined future of the biological system. It is this, in fact, what gives birth to the above strange fact of determined self - maintenance and, as its derived consequence, "self" reproduction, as the symmery attained. It is carried out in a mediated, ,weak" self - reference loop, as a natural prerequisite for the invariance of the genetic code as a „biological invariant of motion”. Being an involved and dubious case, as it is usual, we mainly concentrate on its origin. We suppose a special type of quantum measurement as the original assignation mechanism, with a usual concomitant time inversion symmetry breaking. Symmetry restoring, in fact, is a semiotic control process of the genetic code, the process tentatively conceived to be the living (biological) state of matter.
\end{abstract}

Keywords: The Biological Invariant of Motion, Struggle for Life, Causal Break in Quantum Dynamic, Group Theory, Time Inversion Symmetry Restoring, the Nonlinearity of the Genetic Code

\section{Introduction}

We report here in this study a possible origin, evolution and deeper and also perhaps wider biological function of semiotic controls in biological systems, dealt with in more detail in a previous paper [1]. The issue might be regarded as hypothetical in some respects, still, it might place fundamental molecular biological facts into a different light by outlining an ,unorthodox” origin scenario. It is done by bringing quantum measurement into these highly controversal fields. The argument runs counter to Dawkins' "Selfish Gene" concept, where the author was using a popular terminology, based on data of ethological nature [15]. Our model might perhaps be termed, conforming to this very expressive, if scientifically inaccurate, maybe even misleading, popular terminology, as the similarly „selfish” code one. Dawkin's paradigm cannot be defended on rational grounds: the genetic material soon gets dissolved in the gene pool of the population, so the often frantic, determined fight for its transmission is pointless in the strict sense. Genes are variable entities, which cannot underpin the invariance through geological ages of the similarly invariant phenomenon of "struggle for life”. The latter is meant in the semiotic sense of ,, self" as used here, also the corresponding fundamental "self" - reproduction.

In fact, self - maintainance and, even more, self reproduction are far from being trivial problems, they are experimental facts subject to internal drives, to use an ethological expression, which they do not share with any other purely physical system. Theoretical physicists often refer to life as a "chapter of quantum electrodynamics". We strongly oppose this view. We think that Darwin used the terms „struggle for life” and „reproduction” as in their effects rather hidden empirical facts of a larger scope picture (theory) ${ }^{1}$ [13]. However, without the narrower sense of ,struggle", understood in the above sense of the semiotic „self", evolution would be utterly impossible.

Actually, we think that deciphering theoretically the molecular source of these ethological drives, in their most ancient and primitive forms, present even at the proto organisms, evolutionary theory would be incomplete. In this sense do we refer to the invariance of the genetic code as a basis of the also invariant "struggle for life" in its narrower, struggle of the semiotic „self”, sense.

The „biological invariant of motion”, the genetic code, as 
it is supposed here, would appear as the invariant basis of this „struggle" in the narrower sense (i.e., maintaining the living state). It is, perhaps, a quantum physical „Schrödinger's cat” problem at its ,gedanken experiment's" extreme, were it the case that the outcome depended on the capability (,insight”) of the cat, damaging the setting (thus yielding a pure living state).

In fact, we think that at its core, the popularly sounding term „selfish" code refers to observable facts, as not only the existence, but much more also its action makes it invariant in geological ages, i. e., in the long run. It is just as the ,selfish" gene's existence and, primarily, its action makes itself invariant in a limited number of life cycles, $i$. e., in the short run.

This is, in fact, the phenomenology of our main argument, which is, admittedly, not ethological.

Thus, we think that a brief reference as that above to the scientific core of Dawkins' popular work is in order here, as our tentative alternative paradigm converges to the same phenotypic ethological facts as those of Dawkins', but their molecular roots are radically different in semiotic control terms. This is true in particular since the genetic code assignments are ,virtual”, ,abstract” entities (see [1, 18] and below).

The basic questions, which are not often brought up, rather, usually taken for granted, are: ,why is the pure physical living state of matter so preferred against the also pure physical anorganic state, and why is ,self" reproduction one of the defining characteristics of the former?" Our proposed, perhaps tractable, route is as follows: emphasizing, as it is usual at all similarly dubious facts, their origin.

On this grounds, the basic idea of ours on the origin problem corresponds to a quantum measurement problem, and is fundamentally an introduction into a set of molecular quantum measurements a modified, newly reinterpreted, version of Neumann's analysis of quantum mesurement [2]. This is which was called the ,orthodox" interpretation by Wigner [3], denoted by us as the ,self - distinctioning" paradigm. The reason for the latter expression is presented below in Section 2. 1.. Our corresponding only, but basic, hypothesis is that from protozoa on to humans, they perform „quantum measurements" in this particular sense. We show that for the chemical evolutional emergence of semiotic controls, elementarily the genetic code, a quantum measurement causal break had to come about as it is usual, e. g. [4], but with the following process(es) acting as regressively self - constructing, upbuilding, real - time time inversion symmetry restoring.

We suppose, along this line, that chemical protobiological evolution was a process of an ,evolution of nonlinearity" from nonequilibrium thermodynamics [5] through the ultimate protobiological internal autocatalytic network [6] to the phenotype - mediated nonlinearity of the genetic code [1]. Though we do not provide a detailed stereochemical scenario [7], we hint that the latter had to come about in combination of the seemingly opposite view of the „frozen accident” events [8]. (However, we hint here that as one ,randomly frozen” stereochemical possibility, the „enveloping” protein „devices” at their special conformational amino acid residues as crucial turning points, may have been associated with the proto - RNAs „measured” (ligated) „object”"s similar, complementary conformational turning point ,codons”. This view may be implicite by an inspection of the conformations of $t$ RNAs.)

Furthermore, we define a quantal ,,assignation operator”, in association with the whole internal stable and proper level, supposedly macrocyclic, quasi - causal network, which, by its mapping action, corresponds to the ,subjective mapping” of the Neumannian type. It is related to the experimental measurement outcomes. It is turning the outcome of the borderline membrane (cell - wall) - bound measurement interaction of ,internal" proto - proteins and ,external” proto - RNA oligomers, as separated by a proto - biological membrane (wall) of the ancient vesicule, into some global and persistently maintained ,virtually" entangled, stable states. (We discuss at some length, ,virtuality" in Section 2. 3. and its relation to Bohr - type complementarity in Section 2. 4.). This is a proper basis for the long run, as the genetic assignments (codes) being, as noted above, „abstract”, „virtual” entites (compare Section 2. 3. and a note in [18]). The mappings by the sufficiently evolved internal assignation operator, depending on the material catalytic macrocycle and acting on the material ,measurement outcomes", is the assignation observable of these measurement outcomes. The assignation operator A, as discussed in Sections 2.3., 2. 4, 2. 5., maps the material assignation measurement outcomes onto the whole complementer somewhat diffuse, uncertain ,virtual” internal network with its central ,kernel" (Section 2. 4.), in the primitive conditions of the protobiological systems (Sction 2. 4.), thus it corresponds to Neumann's ,conscious events”.

\section{Discussion}

\subsection{The Orthodox Picture of Measurement and Ancient Genetic Assignments}

The Neumannian version of the „orthodox” measurement interpretation $[2,3]$ is based on the assumption that every physical coupling in the quantum measurement setting, from the quantal object to the brain, obeys quantum dynamics. The final „extra - dynamical”, so „classical” entity, at the end of the series, is consciousness.

We cannot doubt that lower on the evolutional ladder, in lack of a clear evolutional borderline, the organisms similarly ,perform” (quantum) measurements. Thus we are left as origin with the primitive, smooth double layered membrane (cell - wall) - bound (with small, later closing gaps) autocatalytic macrocycle (chemical evolution: see [6]). The most elementary „measurement” then may have been performed by proto - proteins, internally coupled to the macrocycle (compare [9]), as „measurement devices" on external RNA oligomers as quantal „objects”. The effect 
of human consciousness then might have been represented, as noted above, in this ancient case, by an ,assignment operator" (observable) A, which maps the measurement outcome of the above introduced combined stereochemical - constrained frozen accident nature (corresponding to the assignment classes [7]), onto the global - uncertain virtually coherent complementary aspect of the internal polymeric network (Section 2. 4.) (For the measurement reversal of quasi - causality, so of assignations, see below). In this way, the internal mapping events by the central assignment operator mechanism (see below Section 2. 4.) can be regarded as the correspondant of a Neumannian „subjective event" of the vesicule. The mapping mechanism between the material assignment and the virtual space (see Sections 2. 4., 2. 5.) in fact serves as the „Subjective transition component" (brain $\rightarrow$ mind), i. e., in our case to the central ,virtual”, not material internal transition from a subordinated, coordinated set of physical constraints (see e. g. [19]) over the hereby definitely biological material autocatalytic network system. It proceeds to a paralell complementary controlling ,,virtually coherent" existence, setting the control for the very (assignment) constraints (for virtuality, see Sections 2. 3., 2. 4., 2. 5.).

However, the quantum mechanics of the primer measurement setting needs some further elaboration.

We start with a brief communication of Wigner [21]. Wigner showed, that the dynamic interaction of two interacting systems with many degrees of freedom leads to a scattered ,unit" where the scattering is probabilistically distributed among all degrees of freedom, i. e., leads to the occupation of a large portion of the probabilistic state vector space. Without the interaction being „well tailored", it can not be expected that the outcome of the $\mathbf{S}$ matrix scattering out - waves would concentrate with near - unity probability in a very narrow (even single - vector) region of the Hilbert space.

Such a „tailoring" might be brought about e. g. by a measurement constraint. Such a constraint, of course, is not yet specific. Still, there may have been a prior splitting between the autonomous, confined, and dynamical macrocycle and the surroundings, giving rise to a quantum dynamical splitting. It is followed by a dynamical restoring strive, expressible as the splitting up the Universal causality as „observer”, in face of the „observed” outside world, resulting in a so - called ,expectation wavefunction”. Expectation wavefunction $\Psi_{\text {obs }}$ in its original, early conceived, historical sense, was modernized by Heisenberg as „objective tendencies" on the part of the observer [22]. In our terms, this ,expectation wavefunction” is that of the proto - organism, the proto - cell, by absorbing surrounding high energy substrates and building blocks (and excreting waste molecules) as the chemical evolutionary primitive „metabolism”. (Compare e. g. Dyson's construction [16].) (We note here that possibly the most primitive macrocycles utilised directly (also or exclusively) the Sun's energy, but below we deal with, for the sake of simplicity, the probably secondarily arised purely heterotrophic systems.)

Among the molecular objects of the surroundings, there may have been also spontaneously polymerised RNA oligomers, where the steric interaction, by the above hinted mechanism, between codons (,objects”) and amino acid residues („devices”) may have provided specificity. The process may have formed an invariant device - object, if reducible, invariant subspace of the total expectation wave $\Psi_{\text {obs }}$ on the part of the ,observer”. In final terms, the proto - evolutional dimensions of the invariant „outgoing” subspaces could reduce to the primitive genetic code classes, with certain steric relationships between their members.

This process might be called ,self - distinctioning”, i. e., the distinction of the semiotic ,self" and its specific choice of certain molecules from the surroundings (see also below Section 2. 4.).

Note that only a polymer - oligomer overall physical sterically extensive fitting conformations could provide this kind of specificity, containing, as coupling factors, the measurementally sensitive degrees of freedom, i . e., the seeked random but decisive specificity between individual codons and amino acid (residues). It is the seeked crucial specific measurement device - object coupling of the corresponding monomers, their fitting as bolstered, even, if possible, fixing, by the overall loose/rigid gross conformation of the polymeric - oligomeric structural (sterical) frame. This is, in fact, the specific core of the interacting global proto - polymers, the randomly folding proto - proteins and the RNA oligomers in their complementarily „frozen” stereochemistry. This may have lead to ,molecular measurement”.

Besides, there may have been extensively other dynamical interactions, whose usual fate might have been aspecific quantum dynamics (in - out multiwave scatterings, chemical reactions).

Thus the invariant yet subsequently measurementally reducible outgoing Hilbert subspaces in question, the total expectation wavefunction of the „observer" $\Psi_{\text {obs }}$ corresponded to vectors (wavefunctions) as specific, composite, reduced, constrained vectors (functions) of an originally large device - object composite Hilbert space. The process evolves in a measurementally constrained „scattering" way to a more and more specific device object, highly constrained, composite Hilbert vector, a highly constrained outgoing wave, in the long chemical evolution. This was, perhaps, the evolution of a ,well tailored" interaction. Let's not forget that there was a certain part of the very long Pre - Cambrian Age to refine the settings.

The very germ of the above scenario can be found in [9].

According to the very nature of this scenario, which resembles en gross Dyson's two - step scenario of an RNA - invaded internal metabolic network [16], we might term, as noted above, the chain of possible events, from the macromolecular realm on, as a ,self - distinctioning" 
paradigm of quantum measurements. I. e., it might have emerged a distinction (split up) between the internal, causal „observer" systems, primordeal vesicules, and the loosely tightly specific external quantal „objects” (oligomer RNA molecules).

Thus, molecular (and higher level) quantum measurements may have descended evolutionally from molecular quantum dynamics.

\subsection{The Causal Break and Time Inversion Symmetry Restoring}

It can be shown [1] that the external pre - measurement „object" proto - RNA oligomers by the measurements, through the internal ,device” proto - proteins (compare e. g. [10]), entered secondarily from the external world the internal quantally quasi - causal material macrocyclic autocatalytic network. They will act, through the immediate proto-proteins (with quasi - "sharp" coordinates), as intervening quantal, effective ,quasi - causes" in relation to the latter, with the proto - proteins coupled to the internal quasi - ,causal” polymeric network. So there might have emerged a reversed case of the original internal quasi - „cause” (device) proto - proteins and external „effects” (object) measurementally ligated proto - RNAs. They may have been frozen ultimately by the quantal assignation operator A (see below Section 2. 4., 2. 5.).

This overall time reversal process is supposedly due to the splitting up of the symmetric time flow of the internal domain, the above components as sterically largely complementary, but with the internal domain being intrinsically autonomous, intact and quasi - causal. The process thus tentatively follows (derives) from the above noted dynamical splitting between ,observer" and „observed" regions in space.

The splitting up of the symmetry in time may have come about by the en gross internalisation of the „measured", ligated, proto - RNAs as „measured” „objects”, i. e., by the measurement. In other words, with the spatial center of mass, also the moment of the interaction time, as local origins, being on (at) a definite point of the boundary membrane (cell wall), the Hamiltonian of the quantal codon, as conveyed by the ligated $t$ RNA - like ,measured” RNA polymer, and its post - measurement interaction with the proto - protein amino acid residue, in the Schrödinger picture and non - relativistic (Schrödinger) functions, obtains a negative sign in context with the internal macrocycle. The primary cause - effect reversal may have concerned primarily the ,device" proteins, as coupled to the macrocycle, in our local coordinate system. It emerges by the „measured”, ligated RNA entering from the external to the internal context of the protobiological system (with respect to our time - space origins). The internal time flow of the macrocycle becomes, through the coupled proto protein, thus negative (reversed), with the RNAs as ultimate ,causes” in relation to the proto - biological system. Thus there emerges an exceptional globally internally reversed, inversed time process, in which the primary molecular quasi - „causes” are the possibly relatively shorter RNA polymers, conveying the codons, and the primary ,effect” are the proto - protein contained amino acids, evolutionarily coupled globally to the macrocycle. The following ordinary antiunitary internal time inversion operator, containing the post - measurement interactive term,

$$
\mathrm{T}^{-1}=\mathrm{U}^{i / \hbar(\mathrm{H}=\mathrm{H}(\mathrm{RNA})-\mathrm{H}(\text { Prot })+\mathrm{V}(\mathrm{RNA}, \text { Prot, macrocycle })) \tau}
$$

is, however, constrained to introduce to the post measurement interaction pattern, which is in the global internal time inversion process antiunitarily evolving, an other, relative unitary time evolution operator. The latter emerges as the proper, coded proto - proteins interaction with environmental different surrounding organic molecules is corresponding to a time branch back to the RNA molecule(s), in order to produce new proteins, thus forming a loop in time, containing now also a backwards positive time branch.

It is because there comes about a gross, new extra interaction operator, representing the interaction of the RNAs - protein system (and the coupled macrocycle) with its closest environment, representing not too strong, i. e., weak and selected, constrained interactions.

This is, in its essence, not a strictly internal process, and ultimately emerges because the genetic coding function depends nonlinearly on its own coded proteins, e. g. (proto -) ligases, polymerases, and other proto - proteins, and through their effects globally on the whole intact system.

This process then might have corresponded to a secondary internal relative time inversion (a unitary) branch of a partial, highly selective, constrained, open material - semantic loop. It is involving the environment, coupled to indirectly to the genetic mechanism by the loop, and may have given rise thus to a relative unitarity, positive time branch, counter to the original general antiunitarity, $\mathrm{i}$. e. the initial global internal negative time evolution. In this way, the partially, highly selectively open loop involves, as starting and ending points, the genetic code, the two branches of the code $\rightarrow$ environment $\rightarrow$ code process thus evolves internally according to consecutive relative antiunitary and unitary operators without considering the intervening environment.

However, the new, extra interaction operator within the loop, in context with the internal system with reference to the joint internal antiunitary and unitary operators, belonging to the two internal time branches, exhibits not even formally a kind of locally antiunitary - unitary time evolution of this extra interaction operator along the two branches. The environmental interaction operator's time dependence, which is essential to introduce (due to metabolism), is not contained, naturally, by the definition of the time dependence of the Hamiltonians in the Schrödinger picture, i. e., by the antiunitary - unitary internal time evolution operators. What is needed is an $\mathrm{S}$ operator transformation formalism, a transformation from the negative end to the positive one, keeping in mind that 
the overall process is a loop in time. As the new, constrained operator is an interaction energy operator also including the environment, acting at the borderline of the two, internal/external worlds, emphasizedly including the external one, an interaction Hamiltonian is in need, which then can be extended all along the whole loop.

In fact, time dependence is realized by $\mathrm{T}^{*}$ interaction $\left(|\tau|\right.$, (-) $\left.^{\circ} 0\right)$ $\Psi\left(\left(_{(-)} 0\right)_{\text {interaction }} \rightarrow \mathrm{T}^{*}\left(\left(_{(-)} 0,|\tau|\right) \Psi(|\tau|) \rightarrow \Psi\left(_{(+)} 0\right)\right.\right.$ in the interaction picture, which with $\mathrm{T}^{*}$, the time dependent interaction energy operator, describing the time evolution of the whole loop, is a variable entity, depending on the interaction characteristics of the also time dependent wavefunction of the loop. The biological system thus is evolving towards its real - time, regressively predetermined and internally constructed positive time future, time symmetry, in positive internal time $\tau$. The process realizes reccurrently just as the more and more sophisticated is the constructed loop, with the nucleic acids as origins and ends of the process. Here $|\tau|$ is the absolute value of the internal time cycle flow maxima. The action of the two internal relative time reversal (anti)unitarities thus may have been, and contemporarily are, tentatively substituted by a non unitary, partly and selectively open, global time dependent interaction Hamiltonian, a definite, regressively self constructing, positive time evolution in $\tau$. Its general form:

$$
\begin{gathered}
\mathrm{T}^{*}{ }_{\text {interaction }}(\tau)= \\
\mathrm{H}(\tau)_{\text {interaction }}=\begin{array}{c}
\mathrm{V}\left(\tau_{((-)), 1)}\right)_{\text {RNA, Prot }} \rightarrow \mathrm{V}\left(\tau_{(1,2)}\right)_{\text {Prot, }, \text { macrocycle }} \rightarrow \\
\mathrm{V}\left(\tau_{(2,3)}\right)_{\text {macrocycle, environment }}^{\rightarrow}
\end{array} \\
\rightarrow \mathrm{V}\left(\tau_{(3,4)}\right)_{\text {environment, Prot }} \rightarrow \mathrm{V}\left(\tau_{(4,(+) 0)}\right)_{\text {Prot, RNA }}
\end{gathered}
$$

The assignation operator $\mathrm{A}$, depending on the material quasi - causal macrocycle (see Section 2. 4.) maps onto the „virtual" network this reversed assignation. The above mentioned causal break by the quantum measurements [4] is thus eliminated internally from the system: the causal break raised, as an ultimate process, a self - constructing regressive (real - time) time inversion symmetry restoring. The latter is internal to the system, proceeding towards the only available quasi - „causal” time direction, the past. It is building up, ,constructing”, the material proto - cell, in fact, as noted above, towards its regressively predetermed future, in positive time, towards symmetry in time (Section 2. 5.), by the indispensible dynamical interaction with the environment, for the above reasons. Actually, the process may have terminated, and contemporarily is terminating, by a relatively stationary space - mapped time quasi symmetry. We conceive this space - mapped time symmetry as the daughter cells produced by the ancient system, quitting its own individual existence, at least in the elementary, primordial times, also in contemporary cell biology. Contemporarily, there are widely differing forms of ,self" - reproduction, but their roots are the above proto - cellular processes.

We tentatively suppose here that those autocatalytic systems which had not evolved to the required level of internal ,causality”, with an internal autocatalytic network, were quickly eliminated due to fast quantum decoherence, in other words, greatly increased Neumannian entropy. Thus there may have been a strong selection pressure in favor of quasi - causality among the paralell, passively „competing”, intact polymeric macrocyclic networks.

\subsection{Entangled States}

The question arises, how it is possible, that the genetic assignment seems so stable and invariant through the geological ages (compare Section 2. 5.). Also, we would like to investigate here the origin and the consequences of pure physical, other than usual system theoretical, existence of hierarchic controls, of which the genetic code is the most ancient and elementary one, which thus has been termed by us as the ,invariant of biological motion" [1].

We hinted in the Introduction, that the whole living cell, from the outside, from the biologist's viewpoint, can be regarded tentatively as a mutually interdependent, en gross „virtually” coherent, single quantum system (see Section 2. 4.). Even more, the reversedly causal genetic code and its assigned amino acid can be regarded as being kept in a similarly virtual entangled state, inbetween its actions, a distinct, unique quantum system - part of this internal virtual correlated system, with uncertain spatial coordinates (see Section 2. 5.), the former property originating from the molecular quantum measurement via projection operator A (see below and Section 2. 4.). In fact, as it has been shown, the „device” and „object”, i. e., the measurement outcome, inherits from the measurement an entanglement quantum correlation, at least in non demolition experiments [20]. We risk that it is this ,virtual coherence", which we note is a necessary but not sufficient condition, which mediates, rather than destroys in evolution, between its action, in a dynamical mode, this ,virtual” physical relatively strong correlation.

Diagrammatically, there may be a ,virtual' entanglement code/amino acid closed loop, inward running real RNA(/DNA) and real amino acids, and outward running real proteins. During the assignation evolutional process of the code vocabulary, it is the internal systemic quantal assignment operator A (see below) which transforms the post - measurement emerged material code/amino acid measurement outcome, being largely in a sharp spatial correlation (Section 2. 5.), into this virtual dynamical correlation during evolution, with more „uncertain” space coordinates:

with „A" as ,amino acid” and „B” as ,codon”, and $i$ as specific kind of „monomers”, and using the natural composite projection nature of this (virtual) physical correlation,

$$
\rho^{\mathrm{AB}}=\left|\Psi>^{\mathrm{AB}}<\Psi^{\mathrm{AB}}\right|, \mid \Psi>^{\mathrm{AB}} \in H^{\mathrm{A}} \otimes H^{\mathrm{B}}
$$

where $\rho^{\mathrm{AB}}$ is the general density matrix of the entangled system, $\otimes$ indicates the tensor product, the $H$ 's are proper Hilbert spaces (Section 2. 5.); thus we have the special projection 


$$
\begin{aligned}
\left|\Psi_{\mathrm{i}}\left(\mathrm{Q}_{\mathrm{i}}\right)>^{\mathrm{AB}}<\Psi^{\mathrm{iAB}}\left(\mathrm{q}_{\mathrm{i}}\right)\right|\left|\sum_{\mathrm{j}}\right| \Psi_{\mathrm{j}}\left(\mathrm{q}_{\mathrm{j}}\right)>^{\mathrm{AB}} \Rightarrow(\text { const. }) \mid \Psi_{\mathrm{i}} \\
\left(\mathrm{Q}_{\mathrm{i}}\right)>^{\mathrm{AB}} ; \mid \Psi_{\mathrm{i}}\left(\mathrm{Q}_{\mathrm{i}}\right)>^{\mathrm{AB}} \in H^{\mathrm{A} \otimes H^{\mathrm{B}}}
\end{aligned}
$$

with $\left|\Psi^{\mathrm{A}}>=\sum_{\mathrm{i}} \mathrm{c}_{\mathrm{i}}^{\mathrm{A}}\right| \mathrm{i}>_{\mathrm{iA}} \in H^{\mathrm{A}},\left|\Psi^{\mathrm{B}}>=\sum_{\mathrm{j}} \mathrm{c}_{\mathrm{j}}{ }^{\mathrm{B}}\right| \mathrm{j}>_{\mathrm{jB}} \in H^{\mathrm{B}}$ as molecular wavefunctions, indices $i, j$ belonging to different measurement outcomes; so that

$$
\Psi_{\mathrm{i}}^{\mathrm{AB}}=\mathrm{c}_{\mathrm{ii}}\left|\mathrm{i}>_{\mathrm{iA}} \otimes\right| \mathrm{i}>{ }^{\prime}{ }_{\mathrm{iB}}, \text { with } \mathrm{c}_{\mathrm{ii}} \neq \mathrm{c}_{\mathrm{i}} \mathrm{c}_{\mathrm{i}}{ }^{\prime}=1
$$

from where the above introduced nonlinear assignment operator A, ensuring transient assignment invariance by raising the real, sharp material pair to the more diffuse virtual level of the macrocycle (see Section 2. 4.), is defined as

$$
\left(\mathrm{A}=\Psi^{\mathrm{j} A B}\left(\mathrm{Q}_{\mathrm{j}}\right)><\Psi^{\mathrm{j} A B}\left(\mathrm{q}_{\mathrm{j}}\right)\right) \Sigma_{\mathrm{i}}\left(\alpha_{\mathrm{i}}\left(\mathrm{q}_{\mathrm{i}}\right)^{\mathrm{A}}>\beta_{\mathrm{i}}\left(\mathrm{q}_{\mathrm{i}}\right)^{\mathrm{B}}>\right)=\Psi^{\mathrm{j}}
$$

Here Q denotes the global material collective space coordinates (linear combination of local coordinates, including those of the real codon and amino acid), $\mathrm{Q}_{j}$ corresponds to relatively „uncertain” virtual (,symbolically" represented) coordinates (see Section 2. 5.). Assignment operator A, in line with Neumann's analysis, is the observable of the actual assignations, and descends from the material globally ,sharp”, „quasi - causal” macrocycle. The vital projection of Equation (2) is what provides an internally virtually confined (Section 2. 4.), quantum mechanical blueprint selection of the specific proper material assignment histories in the post measurement, internally arising, inverse - time assignation superposition(s) (histories). The latter is the internal post measurement many - to - many manifolds, which, thus, avoid(s) the mixture state, and is specifying (conforming to) the original measurement outcome, as it is subject to the virtual specific "uncertain" assignation (forming „unities”, Section 2. 5.) by the projection constraints of operator A.

On the "reversed" projection, as an ,inverse" of operator $\mathrm{A}$, it is the whole internal polymeric material network, in their coordinates - converted way, materially „projects” out of the assigned material supersymmetric pairs [11, 12] the proper component, in a virtual internal time - series dictated serial way. This process is under the constraints exerted by the (complementer, Sections 2. 4., 2. 5.) virtual pairs, turning the considered material „sharp" nucleotide sequence to a codon unity series. It does it as the pre - set interpretation of the polymeric quantum mechanical base series of material RNA(/DNA). During evolution, on specialisation by evolutional pressure, a separate, well organized „genetic mechanism” of several much more specific polymeric, monomeric and anorganic substances may have been, and contemporarily are, involved, as a serial material expression, realization of the virtual pairs (codons/assigned amino acids).

\subsection{The Material Basis of the Assignation Operator A and Its Function}

Here in lack of corresponding detailed data, we are compelled to make suppositions.

The material holistic organization corresponds to Neumann's subjective wavefunction reduction as the central organizing and self-distinctioning function of the brain, a material basis of the mind. The latter paradigm is evidently an aspect of every measurement, in particular for Neumann's solution. Otherwise, we would have only the smooth unitary time evolution (in the Schrödinger picture).

This self - distinctioning paradigm, however, requires, on the part of the observer, a material internal autonomity and integrity, with a material - semantic closure (Pattee, 1973), say by boundary membranes (walls). This biological fact (break in causality between „observer” and „observed”) might be, actually, the a priori, primary, background break in Universal causality, which logically underlies the quantum measurement causal break.

The internal material quantally ,causal” processes (quantum dynamics), of proto - proteins, fatty acids, carbohydrates and other shorter - longer oligomers, also monomers, embedded into a globally quasi - causal auto and cross - catalytic self - organized network, as noted above, appear to be globally coherent for an outside observer. This is what we termed above often „virtual coherence", a prototype of , $\operatorname{mind}$ ".

The protocells according to our above supposition, may have been complex yet internally globally integrated and autonomous, ,causal” enough (supposing here time to be a parameter) to „perform”, as distincted by their cell membranes (walls), quantum measurements of the Neumannian kind. These were evidently originally ill defined, subject to evolution, as discussed above. It had to take a long time for the quantum dynamics $\rightarrow$ quantum measurement transition.

The emergence of operator A in chemical evolution may have been a material expression, a virtuality function of the measuring, internally integrated, single system, a quasi „causal” global material catalytic macrocycle, a distinct physical quantum mechanical unit (the proto - cell). The latter's measurement capability seems to have appeared by the self - distinctioning paradigm, and was basically set forth by measurement - like interactions on the surroundings, driven by the restoration of the above noted „measuring” part/”object” part splitting.

Operator A thus might have been a mediating entity (an operator, an observable) between the global dynamical material, measuring macrocycle (proto - cell) and its paralell complementary ,virtual coherence”.

Actually, we are here supported by (though later, according to us, erroneously withdrawn) Bohr - type biological complementarity of matter and the (,virtual") living state [24]. Here we apply that form of this complementarity, that e. g. „sharp” material (molecular) space coordinates correspond to more ,uncertain” ,virtual” ones (and on the reverse, discussed in more detail in Section 2. 5.)

The basis is (quantal) „,causality”, by which the quantal „object" of the surroundings becomes part of the internal 
similarly „sharp” quasi - „causal” polymeric network. It strives to restore the decoupled (self - distinctioned), „measuring device"/"object" (proto - cell vs. surroundings) distinction, as measuring proto - enzymes and the surrounding's RNA oligomers (as ,objects”). Thus operator A depends on the underlying matter (proto - proteins, global material network) while, as on the other side of the complementarity, controls (acts on) them as its complementary, virtually coherent, nonlinear, projective observable function (i. e., by the ,virtuality" value of the operator). It therefore forms the nucleotide base groups into an assignation unit (code).

Operator A then may have acted in any measurement, depending on the global material macrocycle and acting on the objects of the surroundings, but evolutionally only the amino acid/codon measurement outcome must have realized, i. e., became evolved enough through evolution into a true molecular measurement, due to the fundamental beneficial controlling effects of the characteristics of the integrated RNAs(/DNAs).

In fact, operator A may have connected the material and virtual sides of the two aspects of the complementarity of the protocell's internal different „causality”. It performed it by mapping the material assignments as measurement outcomes onto the internal, autonomous, integrated, organizing, ill - defined (spatially „uncertain”) „central acausality", the kernel of the interconnected virtual coherence, in a complementary interaction (through operator A). As part of this kernel of the virtual coherence, which may have been corresponding to the conscious mind in Neumann's analysis, it is this indeterminate virtuality, kept through geological ages, which maintains and kept invariant the genetic assignments through evolutionary times inbetween their dynamical actions.

Thus, in fact, as noted above, the material „sharp” global macrocyclic quasi - „causality” manifests itself by „diffuse” virtual coherence, with the measuring, relational operator A as object/device measurement observable (the matter $\rightarrow$ virtuality, corresponding to Neumann's brain $\rightarrow$ mind setting) as an interconnecting, complementarity entity. (Mind, anyway, is also „uncertain” in its „space coordinates".)

Operator A is then such a primordeal internally emerged global material observable, which has emerged on material internal autonomity, integrity and quantal „causality” (,sharp" space coordinates, (Section 2. 5.)), where the whole quantal unit, depending on these "sharp" coordinates, is acting dynamically on the material surrounding ,objects”.

Paralelly, there must have emerged, with its more indeterminate coordinate values, the internal representation of the system, just as its internal virtual coherence, where the latter must have come about before the setting in the external molecular measuremental activity of the proto - cell. I. e., the system was evolving towards the observer/observed splitting. There must have had emerged a central organizing virtual ,kernel" of control above the polymeric network, which was highly ,acausal” and formed (as control) material - semantic closure. The function of the following assignment operator is that it is a specially emerged operator, which conveys fundamental complementarity by ordering the ,sharp” material steric „assignments" (correspondences) to be part of the „uncertain" (and relatively inaccessible) kernel of the virtual coherence of the catalytic macrocycle as virtually maintained codons. The corresponding ,sharp" material „measuring devices" then may have been catalytically active proto - proteins, coupled to the global material "sharp" network.

We are, in summary, lead to the conclusion that complementary ,virtuality”, as we termed the phenomenon here, is a direct prerequisite of any measurement (or proto measurement), by the introduction of which we could use Neumann's interpretation of the body (brain) $\rightarrow$,mind” transition phenomenon in a primordeal scenario. These measurement processes must have been preceded by the pure material „organism” and its „virtual” representation (counterpart) by the „sharp” - „uncertain” co - ordination (Section 2. 5.).

The „kernel” of the macrocycle here, in more details, means the center of the largely self - organizing, largely indefinite (,uncertain”) quantal ,causality”, the kernel of which is en gross ultimately organizing, controllingly interacting with the ,sharp" material auto -, cross catalytic network, ruling the more chaotic, largely unspecific material side processes to make them anyway „causally" closed (with highly „sharp” boundary coordinates).

Of this complementary organization might have emerged in evolution the virtually assigned, but material circular DNA and the protein synthetizing machinery of bacteria. It is executing the virtual assignments as initial semiotic signs (codon "initial symbols”) as a „translational (interpreting) material program", on the material quantum mechanical nucleic acid base sequences.

\subsection{The Physical Basis of the Genetic Code as a "Biological Invariant of Motion"}

\subsubsection{General Considerations}

However complex, the biological system can be regarded as a quantum physical entity, thus does have a Hilbert space and its dual, belonging to strongly interacting molecules, where the Hilbert vectors are time dependent. (For simplicity, we will denote the internal time $\pm \tau$ by $\pm t$.)

Splitting up internally of the inversion symmetry of forward and backward time within the proto - organism, we can suppose inequality of their absolute values of intervals, otherwise the splitting would be inert and arbitrary. The inequality of different time intervals leads to the conclusion that

$$
\text { - } \Delta t=\text { const. } \Delta t,
$$

where const. is not far from unity. 
We also recall the well - known quantum uncertainty principle

$$
\Delta \mathrm{q}_{\mathrm{x}} \Delta \mathrm{p}_{\mathrm{x}}=\hbar,
$$

which also plays a certain role. In this case,

$$
\mathrm{H} \neq \mathrm{H}^{*},
$$

that is, the total Hamiltonian (matrix) will not be equal to its adjoint (in real space symmetric).

(It has its own effect on the concept of ,detailed balance”). It follows that the basic space („ket”space) and its dual, (,bra” space)

$$
\Psi(X, t) \approx \Psi *\left(X^{\prime},-t\right),
$$

and $(-t)$ belongs to the complex conjugate $(*)$ space $\left\{\Psi_{\mathrm{j}}{ }^{*}\right.$ $(X,-t)\}_{\mathrm{j}=1}{ }^{L}(L$ is the number of particles of the system, which, by certain models, can be infinite.) We note it here that they are in fact positive definite (non - trivial metric) spaces, but it does not make a difference in the context of the present paper.

As biology experiences the unique single interconnected and spatially highly structured $\Psi\left(X_{1} \ldots . X_{L}\right)$ wavefunction, in a coordinate complementarity with the $\Psi *\left(X_{1}^{\prime}, X_{L}^{\prime}\right)$ where $X, X^{\prime}$ (denoted by q, Q), correspond to space coordinates (see below). Thus, as $\Psi^{*}$ belongs to $(-t)$, the „bra” wavefunction (vector) set which will represent the local internal time inversion, so foreward time then is represented by the $\left\{\Psi_{\mathrm{j}}\left(X_{1}, \ldots X_{L,}, t\right)\right\}_{\mathrm{j}=1}{ }^{L}$

Our point is that

$$
\sum^{21}{ }_{\mathrm{i}=1, \mathrm{j}} \Delta \mathrm{Q}_{\mathrm{ij}} \Delta \mathrm{q}_{\mathrm{ij}}=\text { const. }
$$

$(\mathrm{Q}=$,virtual”, „bra” space, $\mathrm{q}=$,ket”, material space coordinates, with $i=$ code serial number, $j=$ the coordinates of the rest of the particles of the interacting molecular system $(\mathrm{M}-i), \mathrm{M}=$ number of interacting molecules.)

We suppose, that globally, finally, dynamically, $\mathrm{H}=\mathrm{H}^{*}$ (symmetry attained).

In fact, during the organismic time cycle, the „virtual” $\Psi^{*}(\mathrm{Q})(\mathrm{Q}$ representing, as noted above, the „uncertain”, global space coordinates) with the $L-$ particle entanglement, due in part to the fast - moving „Coulomb holes" of $\mathrm{N}$ electrons in the dynamics, controllingly interact as a projection series, ,program”, on the $\Psi$ (q) „sharp" material space coordinates, involving the $\Psi^{*}\left(\mathrm{Q}_{\mathrm{i}}\right)$ "virtual" units (specific codons of the „virtual” representation.).

This latter is corresponding to the ,inverse” of projection operator A, by the interaction (association) of initially sharp, then, by the (projectional) interaction, uncertain, controlled coordinates of the material molecular groups. They are globally structured enzymes, to form $\mathrm{H}$ - bonds, for example, in a fast moving, diffuse, complicated overturn of matter/energy, i. e., proto - enzymes catalysed chemical reactions, proto - metabolism (Dyson, 1999)). This provides the environment of „uncertain” coordinates for genetic translation.
Thus, with the splitting up of time symmetry, we have a globally, „virtually” controlled dynamical material process. I. e., the "sharp” „bra” vector set, representing time inversion, in an overall way, dynamically builds, acting on the material „ket” set, such a converted organismic (material) „uncertainty” (gross quantal unity), that it is producing, as a final result, by most primordeally quasi space symmetric daughter cells, as (space - mapped) symmetry restored.

The overall material process is but biological experience.

In this way, the „virtual” „bra” set dynamically works to restore the material „ket” space - „bra” - space symmetry $\left(\Psi \sim \Psi^{*}\right)$, where the two total Hamiltonians are equal.

$$
\mathrm{H}^{*}=\mathrm{H}
$$

as $t \sim-t$, so, as a space mapped symmetry,

$$
\mathrm{R} \sim-\mathrm{R},
$$

referring to the daughter cells.

\subsubsection{The Genetic Code}

The primary action of projection operator A is then to turn the material „ket” space, with sharp q

values, already converted by time - inversion a usual „bra” space (the reverse assignation histories) to a time inverted ,, virtual" time - asymmetric globally entangled (in its space coordinates) ,uncertain”, virtual „bra” space.

The assignations, „virtual”, time - asymmeric composite „bra” vectors as they are, are not time invariant in themselves, but via their dynamical control over the "ket” space, as 21 virtual codon unities (the former with a sharp quantum mechanical nucleotide base sequence), converting them to unities with globally more ,uncertain" q' coordinates, and so do they play a central role of progressing towards material (time) symmetry by their dynamical action.

In fact, they do it by interpreting the usual quantum mechanical base sequence of the „ket” space with sharp coordinates (q), converting it to an (,uncertain") material environment, in which the „uncertain” unity (codon) can perform its action.

„Virtual time - invariance" is then a dynamical, progressing one towards real time (space - mapped) time symmetry. The codon is existing individually (detached from the rest of the „virtual” set) in action, where it is directing, directly or indirectly, symmetry - driven, the whole internal material process, towards more and more the recovery of the symmetry, dismissing asymmetry. The dynamical process is the sharp - to - uncertain projection of the genetic code spatial structure by operator A (and on the reverse, by its organismic ,inverse”, the involvement of the cellular machinery with converted material „uncertain” coordinates).

The point is the conversion of nucleotide base groups to „virtually coherent”, single, codon unities, which can carry out, by the reverse process, the task of translation, as the overall dynamical „biological invariant of motion”, the 
base sequence to produce proto - protein synthesis. In fact,

$$
\mathrm{q}_{\mathrm{i}}^{\prime}><\mathrm{Q}_{\mathrm{i}} \mid \mathrm{q}_{\mathrm{i}}>=\mathrm{q}_{\mathrm{i}},
$$

where

$$
\Delta \mathrm{q}^{\prime}{ }_{\mathrm{i}}>\Delta \mathrm{q}_{\mathrm{i}}, \Delta \mathrm{Q}^{\prime}{ }_{\mathrm{i}}<\Delta \mathrm{Q}_{\mathrm{i}},
$$

(the primes meaning the values after the controlling interactions). I. e., the lower grade (more stationary and disjoint) the entanglement, i. e., ,sharper" is $\Psi_{\mathrm{i}}^{*}$ in the „,virtual”, „,inverse" process of projection operator A, the more „uncertain” are the (electronic, nuclear) states of the „ket” space. So does the proto - genetic (translational) machinery become more accurate, dynamically en gross fast, the nucleotide base sequence (coordinates) more readily interpreted by the codon unit wavefunctions.

In this way, however the „biological invariant of motion”, which is representing the usual physical symmetry, which generally does not have a symmetry - induced physical invariant (Wigner, 1959), there might be a (biological) dynamical invariant, (existing only in „ket”, „bra” spaces motion), and time inversion symmetry is attained in cellular (as space - mapped uncertainty) symmetry (division).

Thus, the biological invariant of motion, in strict physical terms, is the invariance of the genetic code as a conserved unit, in the generation - to - generation transmission (dynamics) in geological ages.

Concerning the „uncertain” - „sharp” space coordinate relations, the above proto - enzymatic autocatalytic „kernel” (Section 2. 4. ) and its coupling to the „codon” unity is nonlinear, with global ,uncertain” ,virtual” dynamical coordinates, where $\mathrm{P}$ (gross momenta) is „sharper” and Q is more „uncertain”, in particular in view of the relatively unapproachable nonlinear internal cause effect relations of the central proto - protein autocatalytic cycle. It is this „kernel” - directed primordeal „sharp” molecular metabolism, by taking up energy - rich organic molecules from the surroundings, and giving off waste molecules, which endorses the macrocycle.

Summarising, on the other hand, the material primordeal metabolism turns from "sharp" to ,uncertain” by the organismic ,inverse” of operator A, and the interpretation of the base sequence can be materialised by the „uncertain” $\mathrm{Q}_{\mathrm{i}}$ 's (codon unities).

Generally, P is ,sharp” in an N - electron entanglement on the $\left\{\Psi^{*}\right\}$ virtual basis, thus Q is ,uncertain", and becomes ,sharp" in a controlling interaction with the similarly „sharp" metabolism, turning, as its function, the coordinates of the latter „uncertain”, thus interpreting the base sequence (the genetic code) by the dynamically fast action proto - enzymes.

In an overall way, what is important here is that the biological system is characterized by two, $\Psi^{*}(\mathrm{Q}), \Psi(\mathrm{q})$ single, interacting wavefunctions, which are subject to two relations: the transient conservation of the ,virtual” codon as an unity, in an entangled, coherent environment of the „bra”, $\Psi^{*}(\mathrm{Q})$, wavefunction, and the usual quantum mechanical „sharp” „ket” space, where by the latter there are only nucleotide base sequences. The coordinate „complementarity” is in action, endorsed also by the usual quantum mechanical uncertainty relations between momenta (velocity) and spatial coordinate precision; and makes codon unity interpretation possible in a similarly „uncertain” environment in the „ket” space.

This evolutionally cyclic process might be termed the dynamical invariance of the assignments (the genetic code).

Thus we can quote (borrowing the phrase from a molecular geneticist) that perhaps biology is a dynamical state of matter.

There remains a note on biological experimental verification. The most evident manifestation of the process described above is the biological experimental observation of organisms at higher up on the evolutional ladder. Namely, Haeckel's Law of Ontogenesis. The organism starts from the single fertilized egg, and regressively repeats its past, till arriving the adult (,imago") state, whose sole biological function is ,self" - reproduction.

The primary aim of science is to describe the process under observation. If it is successful, there might come „explanation”, i. e., setting up working models (to quote the Neumannian program). We would be content here to achieve the primary goal.

\subsubsection{The Process of Symmetry Restoring}

The datails of this process provides the biggest challenge in our hypothetical scheme. Tentatively, we have the enzymatic reaction series (see again Pattee, 1974), as internal measurements (e. g. $[17,26,27])$ as $t \sim-t$, so, as a space mapped symmetry,

$$
\mathrm{R} \sim-\mathrm{R}
$$

referring to the daughter cells (steric inversion (quasi - ) symmetry).

What can be said, at this stage of the investigation, in referring to Pattee's concept of an enzyme (chain) of projection operators, that if we decrease the extent of the kernel of the macrocycle, i. e., we restrict ourselves to a minimum cyclic graph of the partially open loop, we know from experimenntal biochemistry, that the process proceeds along enzyme catalysed reactions, where one product is the substrate of the other catalyst. We aim to set up a scheme, where, at the end (the closing step of the graph) leads to, finally,

$$
\begin{aligned}
& \Psi^{*}(-t) \leftrightarrow \Psi(t) \text { (the total Hamiltonian is self-adjoint). } \\
& \Psi_{i}{ }^{\prime}{ }^{\prime}><\Psi_{i}{ }^{*}\left|\Psi_{i}{ }^{S}>\rightarrow \Psi_{i+1} S^{\prime}><\Psi_{i+1}{ }^{*}\right| \Psi_{i}{ }^{\prime}>\rightarrow \\
& \Psi_{\mathrm{i}+1} \mathrm{~S}^{\prime}><\Psi_{\mathrm{i}+2} \mathrm{E}^{*}\left|\Psi_{\mathrm{i}+2} \mathrm{~S}^{\prime}>\underset{\mathrm{S}{ }^{\prime}>}{\Psi_{\mathrm{n}+1}} \rightarrow \rightarrow \Psi_{\mathrm{n}+1} \mathrm{~S}^{\prime}><\Psi_{\mathrm{i}} \mathrm{E}^{*}\right| \\
& \rightarrow \Psi_{n+1}{ }^{\prime}><\Psi_{i}{ }^{\prime *}=\Psi_{i}^{*}{ }^{*} \Psi_{i} \rightarrow\left|\Psi^{*} \Psi\right|^{2} \sim 1 \rightarrow \Psi^{*} \sim \Psi
\end{aligned}
$$

Here $E$ is the corresponding enzyme molecule, $S$ and $S$, are the substrates and product molecules. As it can be seen, the symmetry restoring is purely material, even if ruled by 
the „virtual” states, therefore the symmetry itself is also mapped onto space.

\subsection{The "Weak" Nonlinearity of the Genetic Code and its Strategy as "Biological Invariant of Motion"}

\subsubsection{Struggle of the Semiotic "Self"}

We propose that this overall, basic process belongs to the genetic assignment, the genetic code, which underlies this mechanism. In our hierarchical ordering, permitted by the basic „self - constructing”, upbuilding, time inversion symmetry restoring time evolution, through a series of molecular internal measurements, i. e., through enzyme „catalysed” „biochemical reactions” (e. g. [1], [17]), the following picture emerges: genes are special and secondary configurations of the elementary genetic code. They are formed in order to ensure the invariance of the underlying „abstract" ("virtual”) assignments through the necessarily arising phenotype. The ,aim” of the process is reaching space - mapped time symmetry of the biological system, as the no less strange yet decisive ,self" - reproduction of every living organism. The biological overall process, as pointed out above, is a partially, highly constrained, open, self - constructing semantic - material loop. It evades physical disintegration by the direct internal reverse time process, the reverse chemical evolution, ensuring the virtual entanglement genetic assignments, between and in particular, by their actions, be the invariant of motion in geological ages.

The logical structure of the loop process is ,weak" self reference (nonlinearity), with the phenotype and phenotypic behavior („drives”) forming the external circle of the internally originated loop.

Along this line, it follows that hierarchical informational, semiotic control in general amounts to permanently, reccurrently fixing the (internal/ external) initial conditions of the subsequent, severely constrained internal/ external processes of the organism $[17,23,1]$. The elementary set of the global serial material projections by the virtual codes/assigned amino acids, as noted above, serve as a virtual entanglement - guided interpretation of the pure quantum mechanical RNAs(/DNAs) nucleotide sequence. It acts as a serially gross virtual, ,initial symbol" set in the following recursive process, „program”, of a self constructing real - time material regressive translational algorithm. It is building new, comprehensible sentences out of a set of ,abstract” words, codes/assigned amino acids (compare [18]), i. e., proteins, out of the assigned amino acid ,words”, followed by the phenotype (and phenotypic drives). In this way, the ultimate initial conditions are those of the ,abstract" genetic codes (words) of the RNA(/DNA) polymers, by serially producing new material proteins (,sentences") and, projecting backwards in time to the possible primordeal origin of the contemporary mechanism, as in lack of more evolved specificity, they were proto proteins of the supposedly polymerases class. Possibly, they were which were acting on the RNA(/DNA) polymers (see above Section 2.), as more or less fitting ancient proto
- enzymes.

The strategy of the code is, in other words, probably, involving organisms utilising different energy sources for constructing themselves, i. e., their „futures”, more precisely, later in evolution, specific metabolism, that its task is to maximise ,genetically”, i. e., through passively trial - and - error constructed, ever changes genes, the probability of maintaining its own invariance, constancy, dynamically, in the long run. (Compare ethologically phenotypic cases of e. g. parental care or, extremely, kin selection, presented by Dawkins, all of which also fit our alternative paradigm). E. g. a multicellular predator actively searches, finds and kills its prey in order to constrain the meat to pass down its internal enzyme chains, to produce ATPs (in general). Its main active, ,strategic tool" is its teeth, an evolved ectoderm around the entrance channel of its archenteron. In this way, it fixes the ,initial conditions" of its internal cellular processes, which in turn determine it to repeat the process. The latter is definitely under the control of the genetic material, i. e., in final terms, the genetic code, which is consequently invariant in the dynamical cycles, forming both initial and final states of the processes, even also in evolutional time scales. (The prey animal is generally on a different strategy.) This is its key nonlinearity, whose mechanism is providing initial conditions for the next process.

\subsubsection{Further Studies}

It would be a future task to put the splitting between concrete - material and virtual - control spaces onto a more solid mathematical ground. Perhaps non - trivial metric Hilbert spaces would serve as proper foundings [1] as outlined in Section 2. 5. (This justifies the introduction of the projection expression $\mathrm{Q}_{\mathrm{j}}><\mathrm{q}_{\mathrm{j}}$ in Eqs. (2., 4.)). A kind of product of the supersymmetric state of refs. $[11,12]$ and our dynamical symmetry restoring state would perhaps be a solution. At any rate, we can deduce the framework: introducing the time inversion operator $\mathrm{F}$, in view of the double-stranded DNA, we have

$$
\begin{gathered}
\mathrm{P}\left(\mathrm{t}^{\prime}\right) \rightarrow \mathrm{F}^{-1} \beta_{\mathrm{i}}\left(\mathrm{q}_{\mathrm{i}}\right)><_{\mathrm{i}} \beta\left(\mathrm{q}_{\mathrm{i}}^{\prime}\right)\left(\mathrm{t}^{\prime}{ }_{0, \text { in }}\right) \mathrm{F}=>\alpha_{\mathrm{i}}\left(\mathrm{q}_{\mathrm{i}}\right)><^{\mathrm{i}} \beta\left(\mathrm{Q}_{\mathrm{i}}{ }^{\prime}\right)\left(\mathrm{t}^{\prime}\right) \\
=>\beta_{\mathrm{i}}\left(\mathrm{q}_{\mathrm{i}}\right)><_{\mathrm{i}} \beta\left(\mathrm{q}_{\mathrm{i}}{ }_{\mathrm{i}}\right)\left(\mathrm{t}^{\prime}{ }_{\text {fin }}\right)
\end{gathered}
$$

Here $\beta_{i}>$ is the physical carrier of the codon as quantal nucleotide triplets, while $<^{i} \beta$ is the "abstract semiotic" codon; $\alpha$ is the physical (real) amino acid wavefunction; in and fin denote initial and final states; $t^{\prime}$ is the internal cycle-time flow, while $|-\tau|$, the internal time parameter is defined by $t^{\prime}=|-\tau|-|t|$ with $t$ corresponding to an external time reference. This diagonal element of a composite density matrix, $\mathrm{c}_{\mathrm{ii}} \varphi^{\mathrm{iA}}\left(\mathrm{q}_{\mathrm{i}}\right) \varphi^{\mathrm{iB}}\left(\mathrm{Q}^{\prime}{ }_{\mathrm{i}}\right)$, this "intra-system" correlation function [14] of the evolved code is dynamically invariant under the progressing symmetry restoration and the ultimately attained symmetry, both onto - and phylogenetically. The real invariance of the code is thus presumably under a kind of a product group of $A(5,0) \times F$. 
Concerning experimental verification, the most important context, the virtual entanglement between the genetic code and its assigned amino acid could be assured or refused by ESR or NMR spectroscopy of an in vivo, i. e., from an internal context descended, material loaded $t$ RNA, the correlation's presence or absence in the charge distribution of the two molecular species could be settled, gradually separating them in vacuo while maintaining the gross cellular environment. It can be done in ionized and recombined states of the RNA codon part.

\section{Concluding Remarks}

It has been shown in this report, that there might be presented a fresh view on basic molecular biology, first of all on the genetic mechanism. The central idea was that the genetic code assignments are similarly very stable and invariant in evolution as the similarly very strange, also dynamical persistent stable semiotic "struggle" for existence of the "self" and "self" - reproduction of an in principle purely physical system. We proposed that the key to the problem is the contraction of these seemingly independent facts as to originate from the "abstract" genetic code [18] as a "biological invariant of motion", with its pre - established supersymmetric assignment(s). They act along a regressively self - constructing time inversion symmetry restoring, building regressively, i. e., in positive, real - time, its future (the symmetry), with internally more uncertain, virtually maintained and mediated, hence stable, entangled quantal unity state.

The theoretical origin of the genetic assignment was introduced as having emerged from a primordeal quantum measurement, interpreted according to an "orthodox" version of the concurrent interpretations, actually corresponding basically to the Copenhagen interpretation. The concomitant spontaneous causal break and its internal elimination from the proto - system by a regressive time inversion symmetry restoring split "bra" wavefunction $\left(\Psi^{*}(\mathrm{Q})\right)$, may have given rise to an elementary semiotical, hierarchical control on a pure physical ground. We think that without a causal break of this kind, no real physically based hierarchical semiotic control could have come about. Molecular organizations, by themselves, are not physically hierarchical organizations, descending from (constituted of) their conceptually identical, indistinguishable and so exchanging electrons and identical kinds of nuclei in submolecular quantum theory, which is foreign to hierarchy theory (e. g. [19]).

Concerning symmetry, later on on the evolutional ladder (multicellulars), "self" - reproduction turned to widely different forms, however, elementarily possibly having roots in the space - mapped daughter cells' space (quasi - ) symmetry.

Also, we indicated a possible experimental verification and outlined future theoretical work possibly with the involvement of group theory.

\section{References}

[1] Balázs A (2013) The biological "invariant of motion" vs. "struggle for life"? On the possible quantum mechanical origin and evolution of semiotic controls in biology. Information 4, $367-397$.

[2] Neumann, J (1955) Mathematical Foundations of Quantum Mechanics (Princeton University Press, Princeton), Chapters V. - VI.

[3] Wigner, E P (1961) Remarks on the mind-body question. The Scientist Speculates. ed. Good I.J., (Heinemann, London), pp. 284-302.

[4] Belinfante FJ (1975) Measurement and Time Reversal in Objective Quantum Theory. (Pergamon Press Ltd., Oxford)

[5] Nicolis, G., Prigogine, I. (1977) Self-Organization in NonEquilibrium Systems. (Wiley, New York)

[6] Kauffman S (1993) The Origins of Order. (Oxford University Press, Oxford - New York)

[7] Patel A D (2008) Towards understanding the origin of genetic languages. Quantum Aspects of Life. eds. Abbot D, Davies P, Pati, A, (Imperial College Press, London), Ch. 10. arXiv: $0705.3895 v 2$

[8] Crick F H C (1968) The origin of the genetic code. J. Mol. Biol. 38, 367-379.

[9] Pattee, H H (1971) Can life explain quantum mechanics? Quantum Theory and Beyond ed. Bastin T, (Cambridge Univ. Press, Cambridge), pp. $307-319$.

[10] Pattee, H H (1974) The vital statistics of quantum dynamics. Irreversible Thermodynamics and the Origin of Life. eds. Oster, G.F., Silver, I.L., Tobias, C.A., (Gordon and Breach Science Publishers, New York), pp. 33 - 43.

[11] Bashford, J D, Tsohantjis, I, Jarvis, P D (1997) Codon and nucleotide assignments in a supersymmetric model of the genetic code. Phys. Lett. A. 233, 481-488.

[12] Bashford, J D, Tsohantjis, I, Jarvis, P D (1998) A supersymmetric model for the evolution of the genetic code. Proc. Nat. Acad. Sci. USA 95, 987-992.

[13] Darwin C R (1859) The Origin of Species. (Murray, London)

[14] Elze, H-T, Gambarotta, G., Vallone, F. (2011). Path integral for classical dynamics, entanglement, and JaynesCummings model at the quantum-classical divide. Int. $J$. Quantum Inf. 9, 203-224.

[15] Dawkins R (1976) The Selfish Gene. (Freeman and Co., Oxford)

[16] Dyson F (1999) Origins of Life. (Cambridge Univ. Press, Cambridge)

[17] Matsuno K (1989) Protobiology: Physical Basis of Biology. (CRC Press, Boca Raton)

[18] Patel A D (2001). Quantum algorithms and the genetic code. Pramana - The J. of Phys. 56 (2-3), $367-381$.

[19] Pattee, H H (1973). Physical basis and origin of hierarchical control. Hierarchy Theory, ed. Pattee, H H, (Braziller, New York), Ch. 4. 
28 András Balázs: On the Origin and Invariance of the Genetic Assignments as Elementary Semiotic Controls: A Basic Hypothesis

[20] Zurek W H (2008) Relative states and the environment: einselection, envariance, quantum Darwinism and the existential interpretation. arXiv: 0707.2832v [quant-ph]

[21] Wigner E P (1961) On the probability of the existence of a self - reproducing unit. The Logic of Personal Knowledge: Essays Presented to Michael Polanyi (Routledge and Kegan Paul, London), pp. $231-238$.

[22] Stapp H P (1993) Mind, Matter and Quantum Mechanics (Springer, Berlin - New York)

[23] Popa R (2004) Between Necessity and Probability:
Searching for the Definition and Origin of Life (Springer Verlag, Berlin - Heidelberg)

[24] Bohr N (1934) Atomic Theory and the Description of Nature (Cambridge Univ. Press, London)

[25] Wigner, E. P., 1959. Group Theory and its Application for the Theory of Atomic Spectra, (Academic Press, New York)

[26] Conrad M. (1989) Physics and biology: Towards a unified model. Appl. Math. Comp. 32, 75 - 102.

[27] Conrad M. (1993) The fluctuon model of force, life and computation. Appl. Math. and Comp.56, 208 - 259. 